\title{
Thermal and dynamic mechanical properties of blends of bitumen with metallocene catalyzed polyolefins
}

\author{
Chiara Spadaro - Christopher J. G. Plummer • \\ Jan-Anders E. Månson
}

Received: 5 April 2011/Accepted: 10 June 2011/Published online: 28 June 2011

(C) Springer Science+Business Media, LLC 2011

\begin{abstract}
A high penetration grade bitumen has been blended with up to $50 \mathrm{wt} \%$ of two different grades of metallocene catalyzed linear low density polyethylene (m-LLDPE) in order to investigate the potential of these and similar copolymers as a substitute for styrene butadiene styrene triblock copolymers in polymer-modified bitumens (PMB). A continuous polymer-rich phase was observed at m-LLDPE contents as low as $5-10 \mathrm{wt} \%$, along with a significant decrease in the effective glass transition temperature of the PMBs with increasing polymer concentration, suggesting benefits for low temperature flexibility. The m-LLDPE-based PMBs also showed relatively low dynamic shear viscosities up to high polymer contents in the range of temperature and shear rate corresponding to typical PMB processing conditions. However, the presence of bitumen in the m-LLDPE-rich phase led to a significant reduction in the melting points of the m-LLDPE, and softening of the PMBs at temperatures as low as $40-50{ }^{\circ} \mathrm{C}$, depending on the composition and the melting point of the pure polymer. PMBs based on the m-LLDPE with the higher melting point remained fully elastic in this temperature range, but at the expense of increased crystallinity and a higher glass transition temperature, which limit improvements in low temperature flexibility. On the other hand, the potentially broad composition and property windows associated with m-LLDPEs suggest considerable scope for the fine tuning of PMB properties by using combinations of different m-LLDPEs and/or other polyolefins as a means to optimize performance.
\end{abstract}

C. Spadaro · C. J. G. Plummer ( $₫)$ · J.-A. E. Månson Laboratoire de Technologie des Composites et Polymères (LTC), École Polytechnique Fédérale de Lausanne (EPFL), Station 12, 1015 Lausanne, Switzerland

e-mail: christopher.plummer@epfl.ch

\section{Introduction}

Bitumen is a naturally occurring viscoelastic material whose chemical composition and structure are complex and variable, depending on the source of the crude oil from which it is derived, and on modifications induced by treatments in the refinery or during its service life [1]. The components of bitumen range from saturated hydrocarbons to polynuclear aromatics [2], and it is generally characterized on the basis of "SARA" fractionation in terms of what are termed (i) "saturates," "aromatics," and "resins," referred to collectively as "maltene," and (ii) "asphaltene," the fraction that is insoluble in $n$-heptane [3]. The corresponding microstructures are widely assumed to consist of a colloidal dispersion of micelles of asphaltene in a matrix that consists essentially of maltene, whose average molar mass is relatively low $[1,4,5]$. On the other hand, more recent studies indicate asphaltenes to be soluble in maltenes, so that the concept of a colloidal dispersion and the distinction between asphaltenes and maltenes solely in terms of polarity may be misleading [6, 7].

In applications such as road surfacing and roofing, bitumen is typically blended with polymers in order to improve properties such as flexibility at low temperature, $T$, and high $T$ stiffness, while maintaining weldability and minimizing shrinkage. Polymer-modified bitumens (PMB) may also show improvements in thermal and fatigue cracking behavior, and increased resistance to high $T$ aging [2, 3, 8-14]. The polymer most widely used in PMBs is styrene butadiene styrene (SBS) triblock copolymer, a thermoplastic elastomer in which the polystyrene endblocks act as thermally reversible physical crosslinks and the rubbery polybutadiene mid-blocks provide the elasticity [1]. Although it does not fully dissolve, SBS swells to up to nine times its initial volume when blended with 
Table 1 Physical properties of the polymer modifiers

\begin{tabular}{lllll}
\hline & Comonomers & $T_{\mathrm{g}}\left({ }^{\circ} \mathrm{C}\right)$ & $T_{\mathrm{m}}\left({ }^{\circ} \mathrm{C}\right)$ & $\mathrm{MFI}(\mathrm{g} / 10 \mathrm{~min})$ \\
\hline $\mathrm{PE}-\mathrm{O}$ & Ethylene-octene & -36 & 96 & 30 \\
$\mathrm{PE}-\mathrm{B}$ & Ethylene-butene & -53 & 35 & 5 \\
\hline
\end{tabular}

bitumen, so that even at relatively low SBS concentrations (usually 5-6 wt\%), the swollen polymer may form a continuous phase, significantly modifying the overall properties [2, 5]. Unsaturated elastomers such as SBS are nevertheless susceptible to environmental degradation, particularly under mechanical stress, and are relatively expensive. There is consequently interest in blending bitumen with other polymers, and, in particular, polyolefins, which are available in large quantities at relatively low cost, and show a wide range of mechanical properties depending on the details of their structure [8, 10, 15-27]. However, polyolefins are widely reported to show poor dispersions in bitumen, and PMBs based on such polymers are also prone to morphological instability during storage at elevated $T$, often leading to macroscopic phase separation, and a thick layer of polymer at the blend surface [16, 26, 28, 29]. Metallocene catalyzed linear low density polyethylenes (m-LLDPE) have therefore recently been proposed as alternative modifiers for bitumen, because they combine low cost with improved dispersion characteristics and storage stability with respect to conventional polyolefins [30, 31]. Metallocene catalysis facilitates control of the molecular structure and molar mass distribution, providing polymers with relatively narrow molar mass distributions and a uniform distribution of short chain branches, for example [30, 32, 33]. This permits tuning of bulk properties such as viscosity and crystallinity and may lead to substantially reduced melt elasticity, which has been argued to account for the improved dispersions observed in the corresponding PMBs, since any differences in thermodynamic compatibility with bitumen between m-LLDPEs and other PE-based polymers may be assumed to be minor $[30,31]$. This study focuses on the thermal and dynamic mechanical properties of PMBs prepared from two selected m-LLDPEs with contrasting melt flow indices, MFI, glass transition temperatures, $T_{\mathrm{g}}$, melting temperatures, $T_{\mathrm{m}}$, and degrees of crystallinity, in order to assess their potential for applications that currently make use of SBS-modified bitumens, and to provide pointers for future materials selection.

\section{Experimental}

A high penetration grade bitumen (180/220) was modified by blending with either an ethylene-butene (PE-B) or an ethylene-octene (PE-O) m-LLDPE copolymer $\left(\mathrm{ENGAGE}^{\circledR}\right.$
7447 and ENGAGE ${ }^{\circledR} 8402$, respectively, supplied by Dow Chemical Company) at polymer concentrations ranging from 5 to $50 \mathrm{wt} \%$. The properties of these copolymers are summarized in Table 1. In the blending procedure, which was chosen to simulate industrial processing conditions currently used for bitumen/SBS blends, stainless steel cans with a capacity of approximately $500 \mathrm{~mL}$ were filled with approximately $200-250 \mathrm{~g}$ of bitumen at $80{ }^{\circ} \mathrm{C}$, and the required amount of polymer was gradually added. The cans were closed with a screw top perforated to accommodate a motorized four-blade stainless steel propeller. They were then immersed in an oil bath and $T$ was raised to $180{ }^{\circ} \mathrm{C}$. Each blend was mixed for $2.5 \mathrm{~h}$ at a stirring speed of $60 \mathrm{rpm}$ after which it was removed from the can and left to cool to room $T$ on silicone paper.

For the morphological investigations, the blends were heated and pressed between glass slides, and then observed by optical and fluorescence microscopy using an Olympus AX 70 optical microscope. The application of fluorescence microscopy to PMBs is based on the assumption that the polymer preferentially absorbs the fluorescent constituents of the base bitumen. The bitumen-rich phases thus appear dark in fluorescent light micrographs, while the phases containing the polymer appear light [8]. Image J (US National Institutes of Health) image analysis software was used to determine the projected volume fraction of the polymer-rich regions from the resulting images after suitable thresholding.

Thermal behavior was investigated by differential scanning calorimetry (DSC) using the Q100 from Thermal Analysis Instruments. Capsules containing between 5 and $10 \mathrm{mg}$ of each specimen were subjected to repeated heating and cooling scans under nitrogen between -100 and $200{ }^{\circ} \mathrm{C}$ at a rate of $10 \mathrm{~K} / \mathrm{min}$. Since only minor differences were observed between the first and second heating scans, subsequent discussion will focus on the results from the first heating scans, which were considered to be more representative of the initial mixing conditions.

An ARES rheometer from thermal analysis instruments was used for dynamic shear tests. The plate-plate geometry was used to investigate the PMBs and pure polymers, with a plate diameter of $8-20 \mathrm{~mm}$ depending on the bitumen concentration and hence on the viscosity, and the Couette geometry was used for the unmodified bitumen. In each case, a strain sweep was carried out prior to the dynamic measurements and the dynamic strain amplitude was 
chosen so as to remain within regimes in which the behavior was predominantly linear viscoelastic. $3 \times 1 \times 1 \mathrm{~cm}^{3}$ strips of the PMBs and pure polymers were also tested in dynamic tension using the Q800 dynamic mechanical analyzer from thermal analysis instruments. $T$ was scanned from -50 to $50{ }^{\circ} \mathrm{C}$ at $1 \mathrm{~Hz}$, typically at an applied strain of $0.1 \%$, again in order to obtain a predominantly linear response.

\section{Results and discussion}

A two-phase morphology was observed in all the PMBs, as shown in the fluorescent light images in Fig. 1. PMBs containing $5 \mathrm{wt} \%$ PE-O (Fig. 1a) consisted of swollen polymer-rich inclusions with an average diameter of $20 \mu \mathrm{m}$ dispersed in a continuous bitumen-rich phase. On the other hand, at $5 \mathrm{wt} \%$ PE-B (Fig. 1f), swelling of the polymer was already sufficient to result in phase inversion, the dispersed phase consisting of bitumen-rich inclusions with an approximately constant diameter of $4 \mu \mathrm{m}$. At higher polymer contents, all the PMBs showed a continuous polymer-rich phase and a dispersed bitumen-rich phase. The volume fractions of the polymer-rich phase estimated by image analysis are given in Fig. 2. As the polymer content increased, the volume fraction of the polymer-rich phase also increased and it remained significantly higher than the polymer weight fraction, indicating substantial swelling. Estimates of the corresponding volume fractions, $\phi_{1}$, of bitumen in the polymer-rich phase are given in Fig. 3, obtained by assuming the densities of the different phases to be close to 1 . The bitumen content of the polymer-rich phase decreased with increasing m-LLDPE content and was somewhat greater for PE-O than for PE-B. Moreover, the degrees of swelling at low m-LLDPE contents were comparable to those reported for SBS, i.e., a ninefold increase in volume for an overall m-LLDPE content of $5 \mathrm{wt} \%$, which may be rationalized in terms of the literature values for the three-dimensional Hansen solubility parameters for bitumen [6, 7]; although polyethylenes do not show specific interactions with bitumen, the dispersive components of the solubility parameter, $\delta_{\mathrm{D}}$, for polyethylenes (about 17.5 $\mathrm{MPa}^{1 / 2}$ ) and maltene (17.7 $\mathrm{MPa}^{1 / 2}$ ) are relatively close, implying considerable overlap between their respective solubility spheres [6]. Moreover, asphaltene, as defined by SARA fractionation, is inferred to be far less soluble in polyethylene owing to its stronger dispersive interactions $\left(\delta_{\mathrm{D}}=19.6 \mathrm{MPa}^{1 / 2}\right)$ rather than differences in polarity. Therefore, the heterogeneous microstructures observed in the present case may be considered to result from precipitation of asphaltene-rich domains from a continuous phase composed essentially of maltene and m-LLDPE regardless of whether the asphaltene forms a separate phase in the original bitumen.

Differential scanning calorimetry traces are shown in Figs. 4 and 5 for $\mathrm{PE}-\mathrm{O}$ and $\mathrm{PE}-\mathrm{B}$ and the corresponding PMBs. The DSC trace for pure bitumen showed a broad low $T$ transition comprising two distinct steps in heat capacity, the more marked of these at $-50{ }^{\circ} \mathrm{C}$ generally being attributed components of the maltene fraction [5, 34]. Melting peaks were apparent in both polymer modifiers, but the relatively sharp peak observed for pure PE-O at around $96{ }^{\circ} \mathrm{C}$ suggested a narrower distribution of lamellar thicknesses than for PE-B, which showed a broad peak with a maximum at about $35{ }^{\circ} \mathrm{C}$. The enthalpies of fusion, $\Delta H_{\mathrm{f}}$, of the as-received resins were estimated from the DSC traces to be 113 and $37 \mathrm{~J} / \mathrm{g}$ for PE-O and PE-B, respectively, which correspond to degrees of crystallinity of 40 and $13 \%$ by weight, if the enthalpy of fusion of $100 \%$ crystalline polyethylene homopolymer is taken to be $283 \mathrm{~J} / \mathrm{g}$ [14]. The glass transition in both types of m-LLDPE $\left(T_{\mathrm{g}}=-36\right.$ and $-53{ }^{\circ} \mathrm{C}$ in PE-O and PE-B, respectively) overlapped with the range of $T$ corresponding to the glass transition in the bitumen. As shown in Figs. 4 and 5, addition of the m-LLDPE resulted in a single glass transition, and $T_{\mathrm{g}}$ tended toward that of the pure polymer as the polymer content was increased (Fig. 6).

The $T_{\mathrm{m}}$ of the polymer-rich phase, defined as the value of $T$ corresponding to the maximum in the melting endotherm, were reduced by up to $20 \mathrm{~K}$ with respect to those of the pure polymers, as shown in Fig. 7. The largest decreases in $T_{\mathrm{m}}$ occurred at the lowest polymer concentrations, i.e., at relatively high concentrations of bitumen in the polymer-rich phase (cf. Fig. 3), consistent with previous studies of blends of bitumen with semicrystalline polymers [14, 17, 18, 22]. Such behavior might in principle be accounted for in terms of expressions for the depression of $T_{\mathrm{m}}$ of a semicrystalline polymer in the presence of a low molar mass solvent derived from the Flory-Huggins theory for the thermodynamics of polymer-solvent mixtures. For low $\phi_{1}$, this leads to:

$\frac{1}{T_{\mathrm{m}}}-\frac{1}{T_{\mathrm{m}}^{0}}=\frac{R V_{\mathrm{pu}}}{V_{1} \Delta H_{\mathrm{pu}}}\left(\phi_{1}-\chi \phi_{1}^{2}\right)$

where $T_{\mathrm{m}}^{0}$ is the melting point of the pure polymer, $V_{\mathrm{pu}}$ is the molar segmental volume of the polymer, $V_{1}$ is the molar volume of the solvent, $\Delta H_{\mathrm{pu}}$ is the molar enthalpy of crystallization per segment of the pure polymer (taken to be $4100 \mathrm{~J} / \mathrm{CH}_{2}$ [14]), and $\chi$ is the Flory-Huggins interaction parameter [35]. In the present case of a heterogeneous solvent, whose composition is also expected to vary with its concentration in the polymer, $V_{1}$ and $\chi$ are poorly defined. However, assuming $\chi$ to be of the order of 0.5 , Eq. 1 provides a reasonable fit to the variation in $T_{\mathrm{m}}$ with $\phi_{1}$ for both polymers at low $\phi_{1}$ implied by Fig. 7 for 
Fig. 1 Fluorescence

micrographs of m-LLDPE modified bitumen: $\mathrm{PE}-\mathrm{O}$ :

a $5 \mathrm{wt} \%$, b $10 \mathrm{wt} \%$, c $20 \mathrm{wt} \%$, d $30 \mathrm{wt} \%$, e $50 \mathrm{wt} \%$; PE-B:

f $5 \mathrm{wt} \%$, g $10 \mathrm{wt} \%$, h $20 \mathrm{wt} \%$, i $30 \mathrm{wt} \%$, j $50 \mathrm{wt} \%$
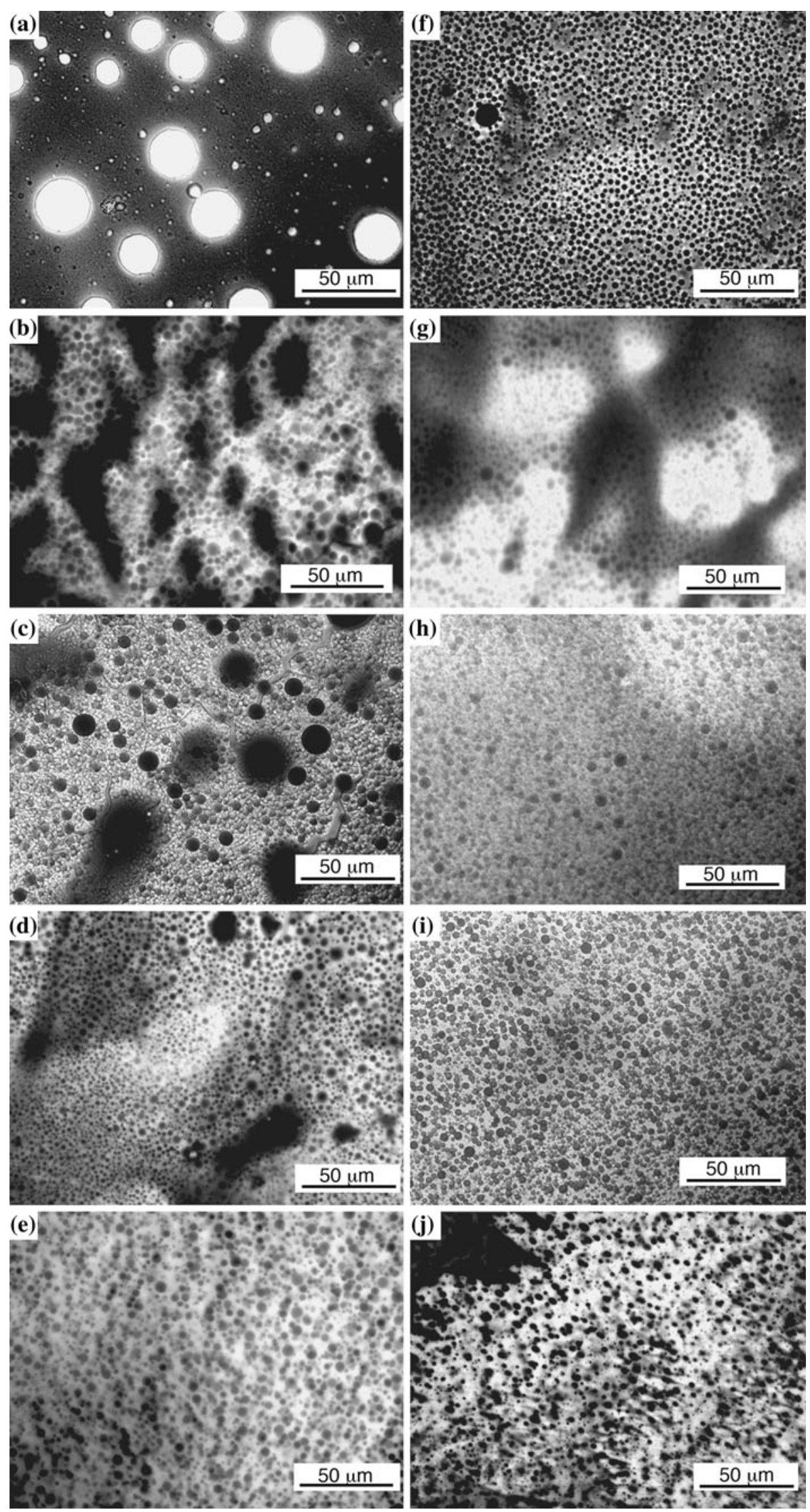


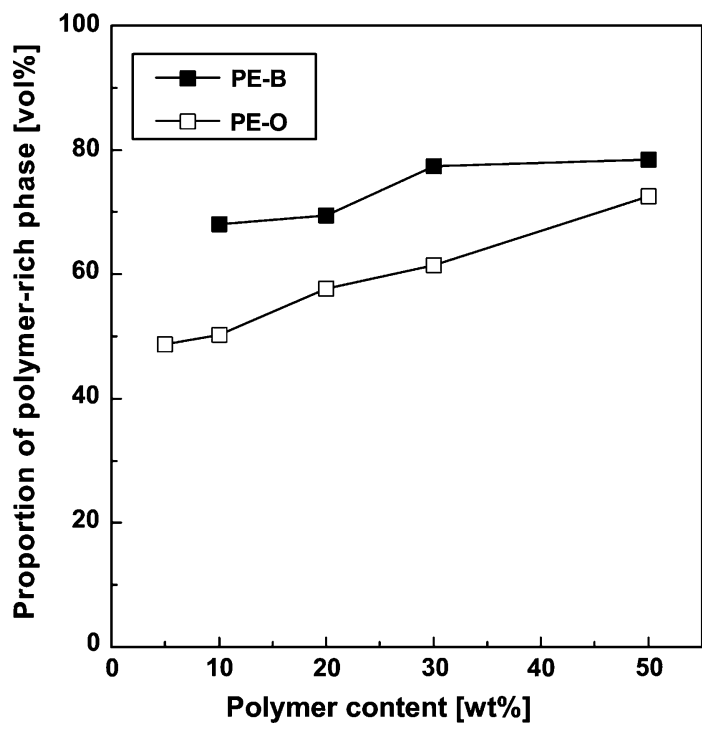

Fig. 2 Volume fraction of the polymer-rich phase in the PMBs as a function of the polymer content

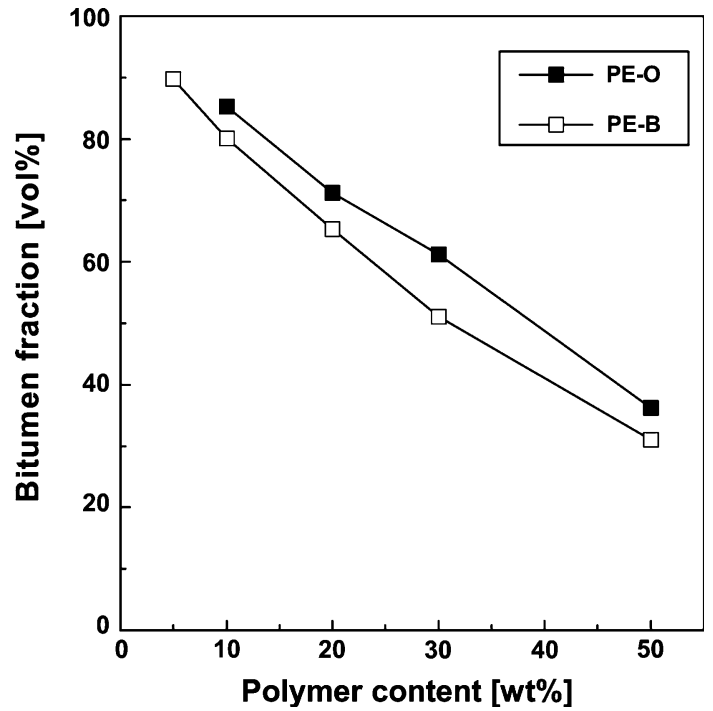

Fig. 3 Bitumen content in the polymer-rich phase as a function of the overall polymer content of the PMBs

$V_{\mathrm{pu}} / V_{1}=0.2$. Bearing in mind the limitations of the FloryHuggins approach in the present context, this suggests similar affinities between the two different m-LLDPE and the bitumen. Hence, the greater solubility of the maltene in $\mathrm{PE}-\mathrm{O}$ inferred, e.g., from Fig. 3, may be a consequence of the higher MFI of this latter (which implies a lower average molar mass).

The variation of $\Delta H_{\mathrm{f}}$ with composition estimated from the DSC traces for the PMBs is shown in Fig. 8. In each case, $\Delta H_{\mathrm{f}}$ increased roughly monotonically with increasing polymer content (absolute values were difficult to determine with precision owing to uncertainties in the baseline, particularly in the case of the PE-B-based PMBs, in which

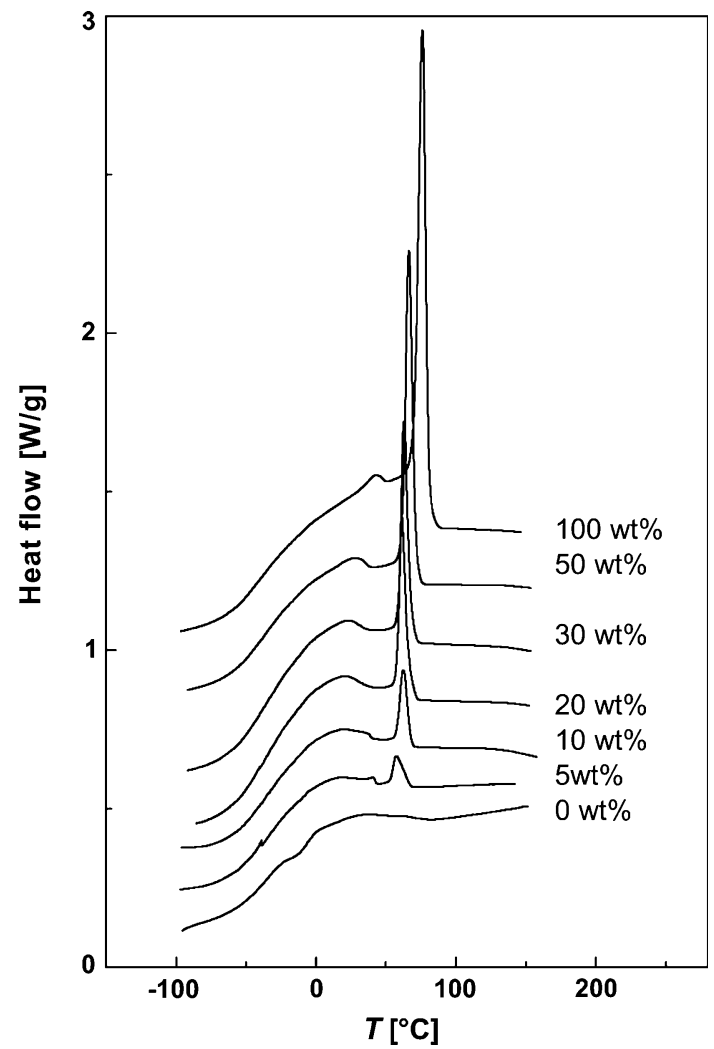

Fig. 4 DSC heating scans for pure bitumen and PE-O modified bitumen

the melting transition overlapped with the glass transition; in future study, it would therefore be of interest to use modulated DSC, which allows one to separate reversing and non-reversing components of the heat flow signal and hence deconvolute melting peaks and glass transitions under appropriate scanning conditions). However, the increases in $\Delta H_{\mathrm{f}}$ with polymer content appeared greater at low polymer contents than would be expected on the basis of simple proportionality with the polymer content, indicating increased crystallinity in the presence of the bitumen and/or co-crystallization of part of the bitumen. Similar trends were observed by Fawcett et al. [17], who found that PMBs containing LLDPE with a relatively high degree of crystallinity showed further increases in the apparent degree of crystallinity of the LLDPE with decreasing polymer content, although they reported the opposite behavior for a less crystalline LDPE.

Tensile storage moduli $\left(E^{\prime}\right)$ and loss angle $(\tan \delta)$ of the PMBs and pure polymers are shown in Figs. 9 and 10. The tests were carried out at $T$ up to $50{ }^{\circ} \mathrm{C}$, beyond which, all the materials became too soft to allow precise determination of the tensile stress in the linear viscoelastic regime. Even under these conditions, satisfactory measurements were only possible for PMBs with relatively high polymer contents. As shown in Fig. 9, $E^{\prime}$ for the pure PE-B 


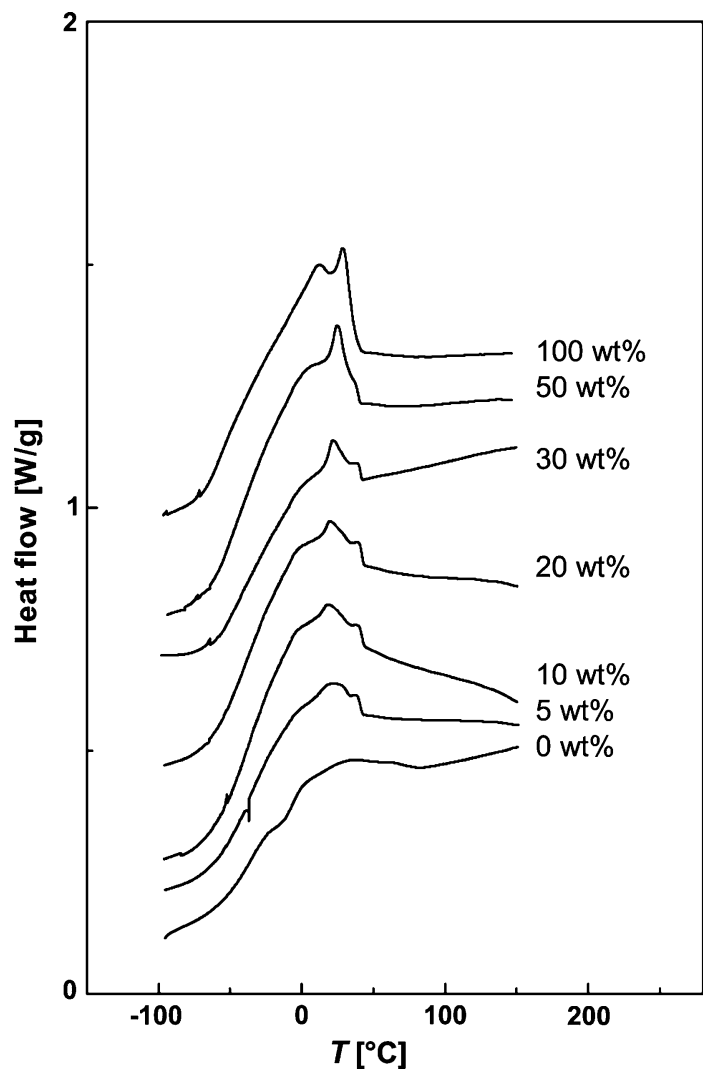

Fig. 5 DSC heating scans for pure bitumen and PE-B modified bitumen

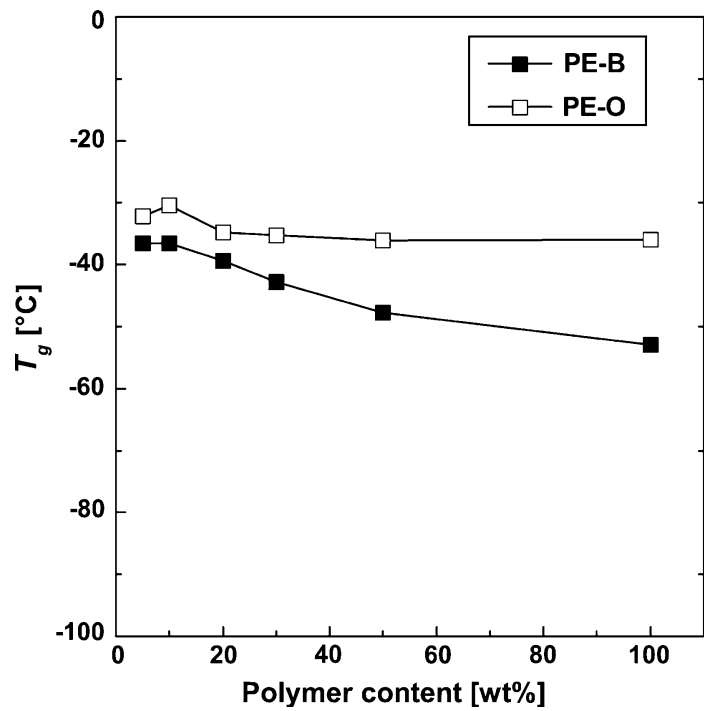

Fig. 6 Glass transition temperature, $T_{\mathrm{g}}$, as a function of polymer content for the different PMBs

decreased by about two orders of magnitude over the range of $T$ investigated, and a rubbery plateau was present between approximately -20 and $30{ }^{\circ} \mathrm{C}$, melting leading to a sharp drop in $E^{\prime}$ at higher $T$. At very low $T$, there was also a suggestion of a second plateau corresponding to the

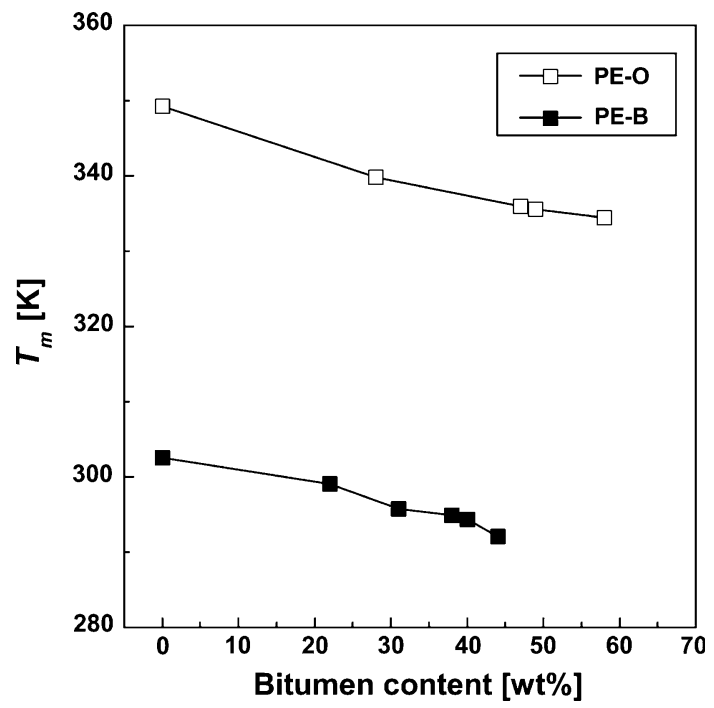

Fig. 7 Melting temperature, $T_{\mathrm{m}}$, as a function of bitumen content in the polymer-rich phase for the different PMBs

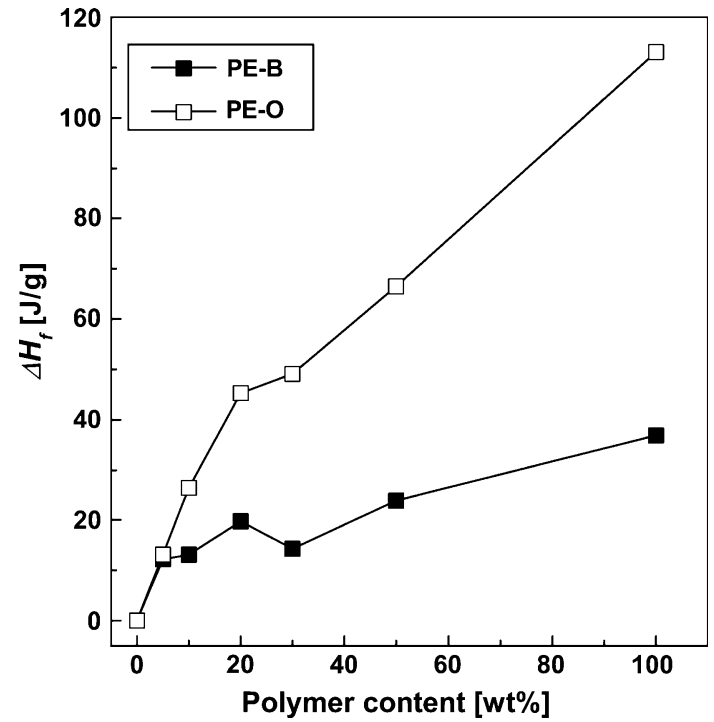

Fig. 8 Heat of fusion, $\Delta H_{\mathrm{f}}$, for the different PMBs

glassy regime below $T_{\mathrm{g}}$. As the bitumen content increased in the PMBs containing PE-B, the rubbery plateau became restricted to $T$ immediately below $T_{\mathrm{m}}$. Moreover, $E^{\prime}$ showed a monotonic increase with bitumen content at low $T$, so that at $-50{ }^{\circ} \mathrm{C}, E^{\prime}$ for the PMB containing $20 \mathrm{wt} \%$ polymer was about one order of magnitude greater than that of the pure polymer, consistent with the higher low $T$ stiffness of the bitumen in this case. However, the curves for the different materials converged as $T$ increased. As also shown in Fig. 9, there was a broad peak in $\tan \delta$ centered at about $-50{ }^{\circ} \mathrm{C}$ in the pure $\mathrm{PE}-\mathrm{B}$, corresponding to the $\gamma$-transition, which is generally associated with the glass transition, consistent with the DSC results [36]. This low $T$ peak shifted to higher $T$ as the bitumen content 


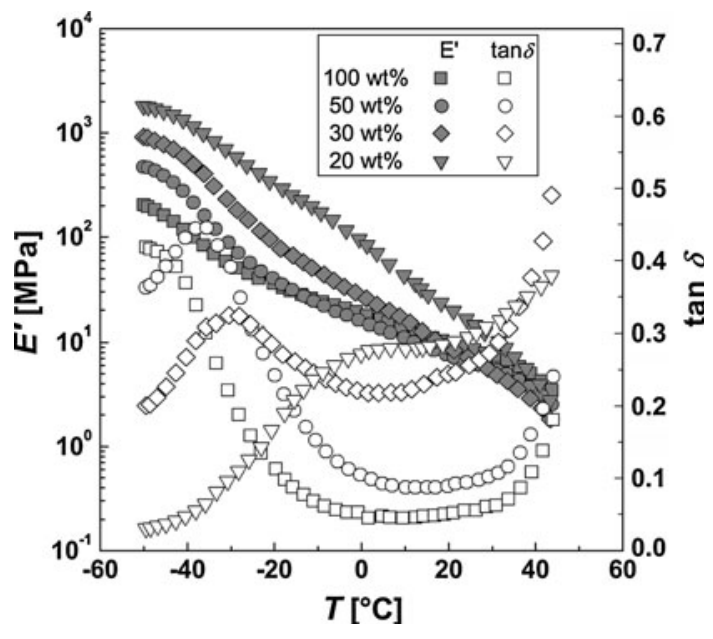

Fig. 9 Variation in tensile storage modulus $E^{\prime}$ and in the loss angle, $\tan \delta$, with composition for PE-B and PMBs containing PE-B

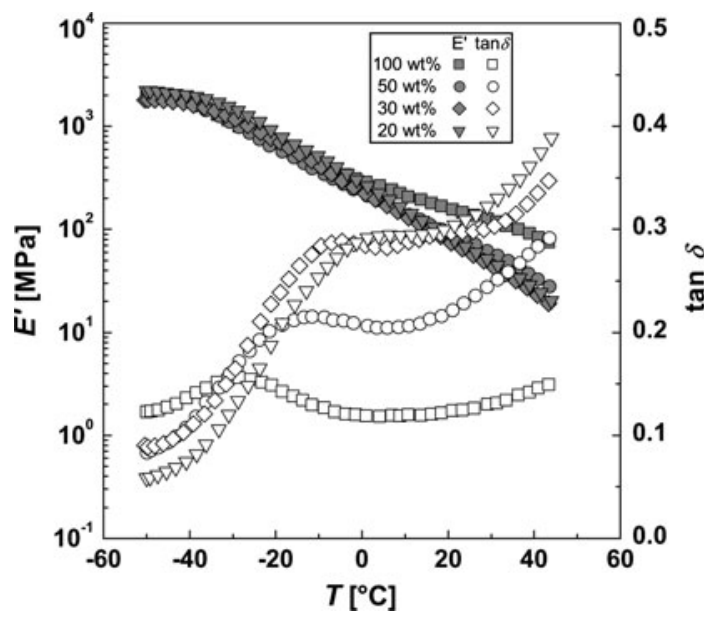

Fig. 10 Variation in tensile storage modulus $E^{\prime}$ and in the loss angle, $\tan \delta$, with composition for $\mathrm{PE}-\mathrm{O}$ and $\mathrm{PMBs}$ containing $\mathrm{PE}-\mathrm{O}$

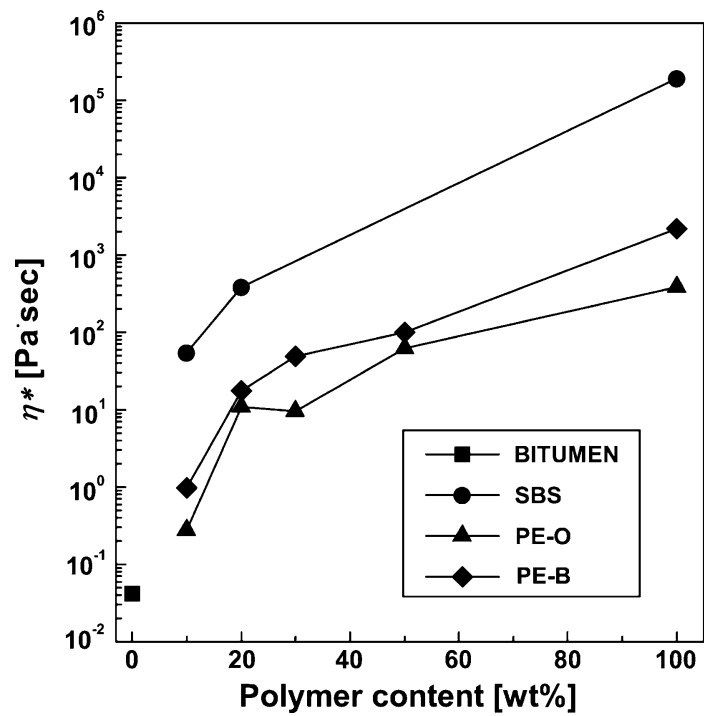

Fig. 11 Complex viscosity, $\eta^{*}$, as measured at $180{ }^{\circ} \mathrm{C}$ for pure bitumen and different PMBs

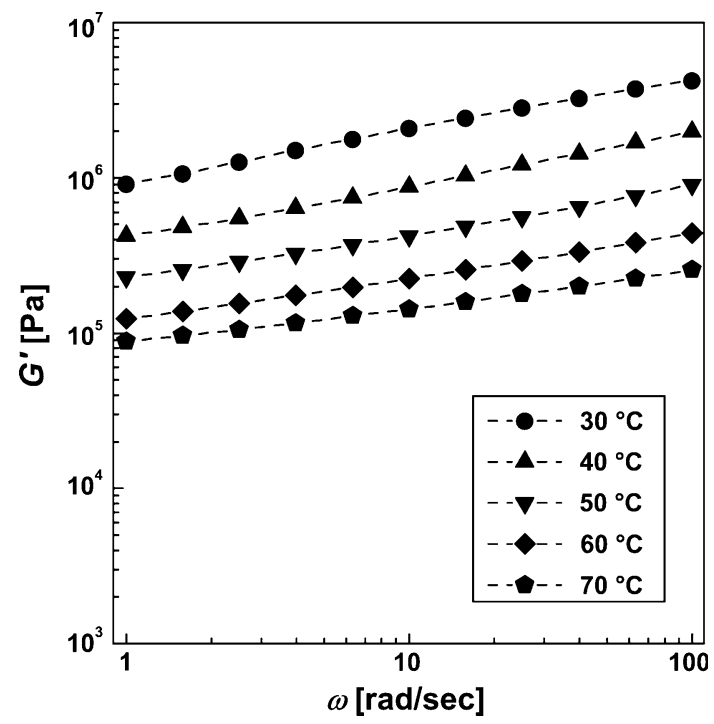

Fig. 12 Shear storage modulus, $G^{\prime}$, of $20 \mathrm{wt} \%$ PE-O PMB at different $T$

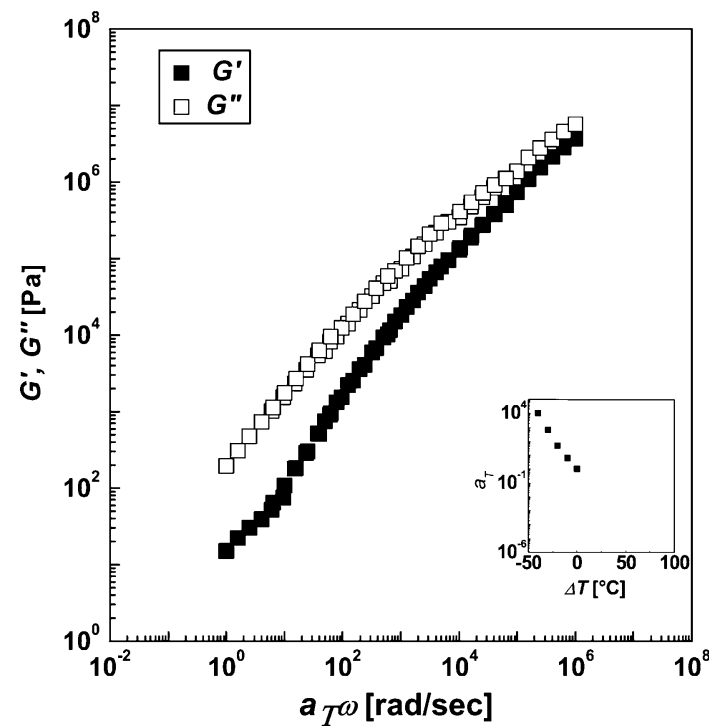

Fig. 13 Master curves of the dynamic viscoelastic functions of the unmodified bitumen at a reference temperature $T_{\mathrm{r}}=50^{\circ} \mathrm{C}$. The inset shows the corresponding shift factors, $a_{T}$, as a function of $\Delta T=T-T_{\mathrm{r}}$

increased. Data for $E^{\prime}$ and $\tan \delta$ are given in Fig. 10 for $\mathrm{PE}-\mathrm{O}$ and the corresponding PMBs. The curves for $E^{\prime}$ were very close for all the PMBs based on $\mathrm{PE}-\mathrm{O}$ at low $T$, reflecting the relatively high degree of crystallinity, high $T_{\mathrm{g}}$ and hence high stiffness of the PE-O at low $T$. Indeed, increasing the bitumen content led to a decrease in $E^{\prime}$ at high $T$. As with PE-B, PE-O showed a broad peak in $\tan \delta$ corresponding to the $\gamma$-transition, which again shifted to slightly higher $T$ as the bitumen content increased. 
Fig. 14 Master curves of the dynamic viscoelastic functions of PMBs containing PE-B at a reference temperature $T_{\mathrm{r}}=50{ }^{\circ} \mathrm{C}$. The inset shows the corresponding shift factors, $a_{T}$, as a function of $\Delta T=T-T_{\mathrm{r}}$
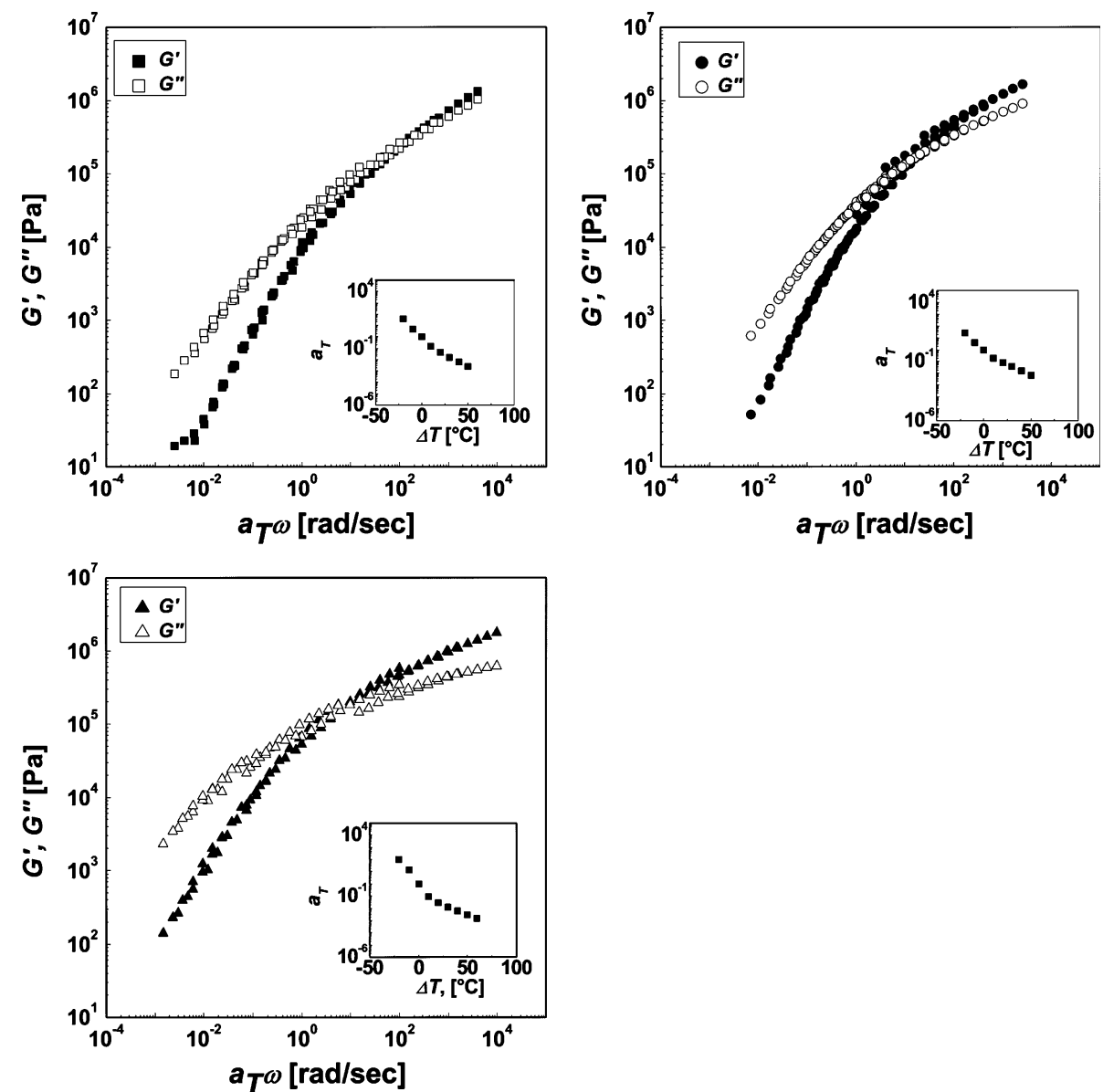

Figure 11 shows the magnitude of the complex viscosity, $\eta^{*}$, of the PMBs and pure polymers at the mixing temperature of $180{ }^{\circ} \mathrm{C}$ and a frequency of $1 \mathrm{~Hz}$, along with results from blends modified with SBS copolymer for comparison. Regardless of the type of polymer additive, the PMBs showed significantly higher viscosity than the unmodified bitumen. However, the viscosity increases were far greater for the specific grade of SBS investigated here (C-311 from Repsol ${ }^{\circledR}$ ) than for both types of m-LLDPE, reflecting the high viscosity of the pure SBS, so that the viscosity of a PMB containing $10 \mathrm{wt} \%$ SBS was comparable with a PMB containing $50 \mathrm{wt} \%$ PE-B. Indeed, the viscosity of the pure $\mathrm{PE}-\mathrm{O}$ was less than an order of magnitude greater than the PMB containing $10 \mathrm{wt} \%$ SBS.

Figure 12 shows a representative example of the evolution of the dynamic shear storage modulus, $G^{\prime}$, in the PMBs as a function of frequency at different $T$ (in this case for a PMB containing $20 \mathrm{wt} \%$ PE-O). $G^{\prime}$ increased monotonically with both frequency and $T$ in all the materials, and, as suggested by Fig. 12, the data could generally be superposed by lateral shifts along the $T$ axis. Timetemperature superposition was therefore used to provide an indication of the response at a reference temperature of $50{ }^{\circ} \mathrm{C}$ (representative of the upper range of service temperatures) over an extended frequency range, as shown in Figs. 13, 14, 15, where master curves for $G^{\prime}$ and the loss modulus, $G^{\prime \prime}$, are given as a function of frequency for pure bitumen and the PMBs containing up to $30 \mathrm{wt} \%$ m-LLDPE, along with the corresponding shift factors, $a_{T}(T)$. The data showed reasonable overlap in this composition range, but it should be emphasized that bitumen is known to be a thermo-rheologically complex material, as discussed extensively elsewhere, so that the absolute frequency scale should be treated with caution [24, 37, 38]. On the other hand, the $a_{T}(T)$ were similar for the different PMBs, so that the master curves provided a convenient means of comparing their overall behavior.

The unmodified bitumen showed a predominantly viscous response (Fig. 13), the loss modulus $G^{\prime \prime}$ exceeding the storage modulus $G^{\prime}$ at all the frequencies and $T$ investigated, although the curves tended to converge at the highest frequencies/lowest $T$, consistent with previous observations [30]. The PMBs containing PE-B also showed a viscous response at low frequencies (Fig. 14), with $G^{\prime}$ and $G^{\prime \prime}$ proportional to $\omega^{-1}$ and $\omega^{-2}$, respectively in the limit $\omega \rightarrow 0$, consistent with the expected behavior for a linear polymer melt in the terminal zone. However, as the frequency increased and/or $T$ decreased there was a 
Fig. 15 Master curves of the dynamic viscoelastic functions of PMBs containing PE-O at a reference temperature $T_{\mathrm{r}}=50{ }^{\circ} \mathrm{C}$. The inset shows the corresponding shift factors, $a_{T}$, as a function of $\Delta T=T-T_{\mathrm{r}}$
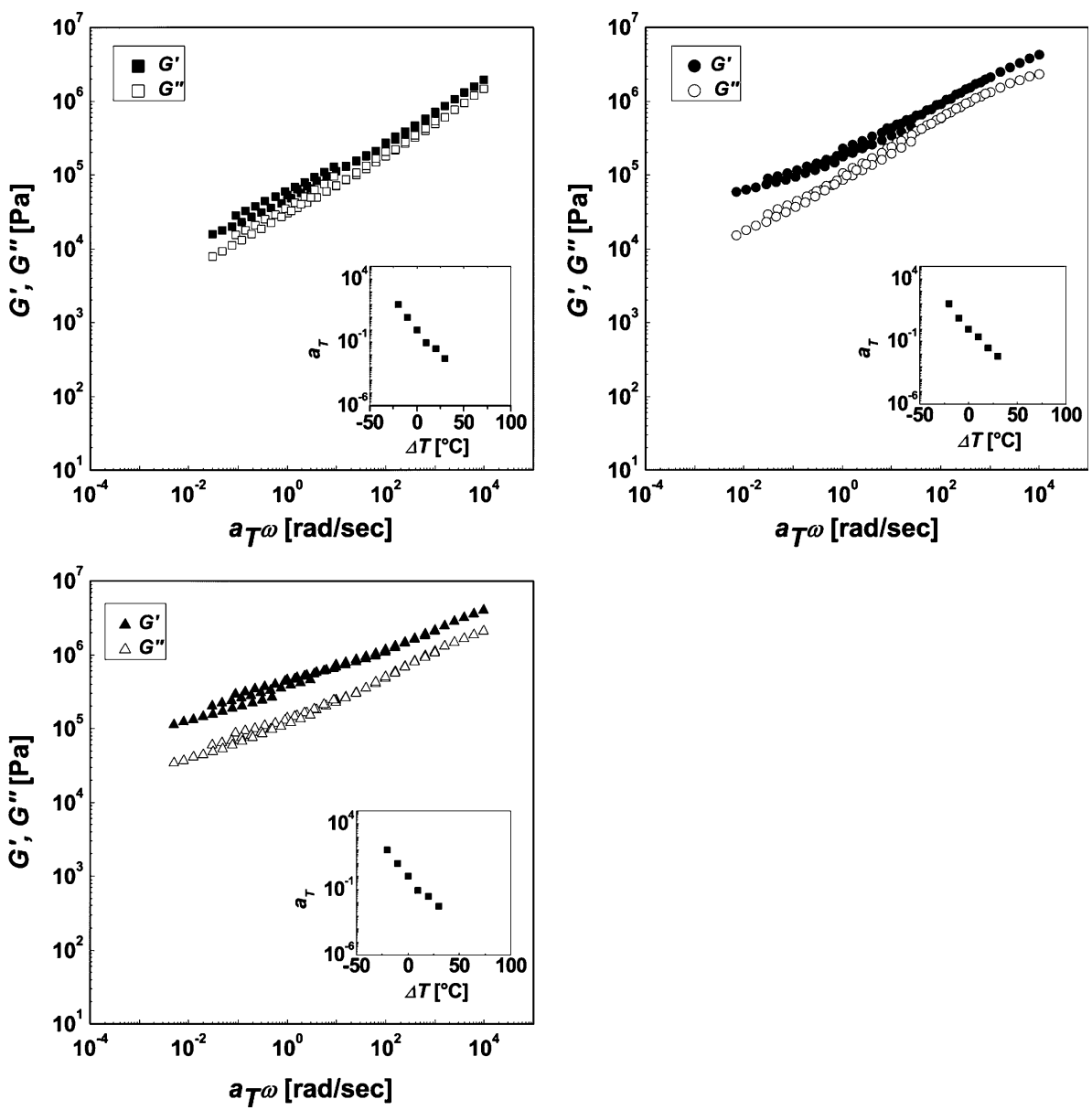

crossover between $G^{\prime}$ and $G^{\prime \prime}$, which became more apparent at higher polymer contents, reflecting an increasingly elastic response. The apparent crossover frequency, $\omega_{\mathrm{c}}$, at the reference temperature of $50{ }^{\circ} \mathrm{C}$ used for the superposition, and the corresponding values of $G^{\prime}$ and $G^{\prime}$, did not change markedly with PE-B content, although $\omega_{\mathrm{c}}$ did increase somewhat as the PE-B content was reduced from 20 to $10 \mathrm{wt} \%$. A similar crossover has been reported in other polyolefin-based PMBs, along with an increase in $\omega_{\mathrm{c}}$ for the PMBs with respect to that observed in the pure polymer, which has been attributed to the plasticizing effect of the bitumen [30]. In the case of the PE-O-based PMBs (Fig. 15), $G^{\prime}$ remained greater than $G^{\prime \prime}$ over the entire frequency range, even at the lowest $\mathrm{PE}-\mathrm{O}$ contents, indicating predominantly elastic behavior, although the differences in $G^{\prime}$ and $G^{\prime \prime}$ increased as the polymer content increased. Moreover, the frequency dependence of $G^{\prime}$ was also relatively weak in these materials, implying improved long-term mechanical stability at $T$ in the vicinity of $50{ }^{\circ} \mathrm{C}$.

These initial results for m-LLDPE-based PMBs are promising in that they indicate similar compatibility with the bitumen to that observed for SBS, in spite of the significant differences in polarity between SBS and m-LLDPE, which is important for the morphological stability, and they also confirm that relatively good dispersions may be obtained under standard processing conditions. Significant improvements in low $T$ flexibility have been observed, the PMBs containing PE-B, in particular, showing significantly reduced stiffness down to $T$ in the range of $-50{ }^{\circ} \mathrm{C}$, whereas $\mathrm{PMBs}$ containing $\mathrm{SBS}$ tend to harden below $-30{ }^{\circ} \mathrm{C}$ [39]. Moreover, dynamic viscosity measurements showed that it should be possible to envisage a relatively wide range of compositions without substantial modification to mixing and processing procedures currently used for SBS, which has the disadvantage of being more expensive and more sensitive to UV exposure.

High $T$ stability, nevertheless, remains a concern with the m-LLDPEs considered in this study, owing to the relatively low $T_{\mathrm{m}}$ of the m-LLDPE-rich phase, the onset of melting being evident from the substantial decreases in $E^{\prime}$ from $T$ as low as $40{ }^{\circ} \mathrm{C}$ in the PE-B-based PMBs (Fig. 9), which is incompatible with industrial criteria for outdoor applications (PMBs containing SBS show a rubbery plateau that extends up to about $80{ }^{\circ} \mathrm{C}$ ). The melting onset in pure PE-O occurred at significantly higher $T$ (cf. Table 1), but there remained a significant loss in stiffness for $T>40{ }^{\circ} \mathrm{C}$ in the PE-O-based PMBs. These latter continued to show essentially elastic behavior in this range of $T$, 
implying improved high $T$ stability, albeit at the expense of increased crystallinity and low $T$ stiffness, but it is clear that even in this case, the high $T$ response must be improved if practical implementation is to be envisaged. It may nevertheless be possible to exploit the relatively good miscibility of m-LLDPEs with other polyolefins (other LLPDEs or LDPE) in order to tailor the high $T$ response of the polymer modifier and hence the melting behavior of the $\mathrm{PMB}$, and the results also imply considerable scope for controlling the low $T$ response by varying the degree of crystallinity and $T_{\mathrm{g}}$. This will provide the focus for the next stage of the study, in which a compromise will be sought between high $T$ stability and low $T$ elasticity, in order to provide a material that corresponds to current industrial norms.

\section{Conclusions}

The two types of m-LLDPE investigated in this study as additives for PMBs show some promising characteristics, notably reduced low $T$ stiffness with respect to PMBs modified with SBS and relatively low viscosities up to high polymer contents in the range of $T$ corresponding to typical PMB processing temperatures. Fluorescence microscopy showed a continuous polymer-rich phase to be present in the m-LLDPEs over most of the composition range investigated, indicating a high degree of solubility of the bitumen in the respective polymers. However, the presence of the bitumen led to a significant reduction in $T_{\mathrm{m}}$ in the polymer-rich phase, and hence excessive softening of the PMBs based on a relatively low $T_{\mathrm{m}}$ m-LLDPE for $T$ exceeding 40 to $50{ }^{\circ} \mathrm{C}$. Blending with a higher $T_{\mathrm{m}}$ m-LLDPE led to better high temperature stability, but, in the present case, this was accompanied by a higher degree of crystallinity and a higher $T_{\mathrm{g}}$, which may limit improvements in low $T$ flexibility. These results therefore suggest that the overall properties may need to be tailored by blending different m-LLDPE and/or other compatible polyolefins in order to achieve an optimum compromise between high and low $T$ performance.

Acknowledgement The authors wish to acknowledge the financial support of the Swiss Innovation Promotion Association (KTI/CTI).

\section{References}

1. Airey GD (2004) J Mater Sci 39:951. doi:10.1023/B:JMSC. 0000012927.00747 .83
2. Isacsson U, Lu X (1995) Mater Struct 28:139

3. Read J, Whiteoak D (2003) The Shell bitumen handbook, 5th edn. Thomas Telford, London

4. Loeber L, Muller G, Morel J, Sutton O (1998) Fuel 77:1443

5. Lesueur D (2009) Adv Colloid Interface Sci 145:42

6. Redelius PG (2004) Energy Fuels 18:1087

7. Hansen CM (2007) Hansen solubility parameters: a user's handbook, 2nd edn. CRC Press, Boca Raton

8. Ait-Kadi A, Brahimi B, Bousmina M (1996) Polym Eng Sci $36: 1724$

9. Lu X, Isacsson U (2001) Polym Test 20:77

10. Airey GD (2002) Construct Build Mater 16(8):473

11. Yildirim Y (2007) Construct Build Mater 21:66

12. Fawcett AH, Lor SK (1992) Polymer 33(9):2003

13. Oba K, Bjork F (1993) Polym Test 12:35

14. Fawcett AH, McNally T, McNally GM, Andrews F, Clarke J (1999) Polymer 40:6337

15. Oba K, Roller A (1995) Mater Struct 28:596

16. Giavarini C, De Filippis P, Santarelli ML, Scarsella M (1996) Fuel 75:681

17. Fawcett AH, McNally T, McNally GM (1999) J Elastom Plast $31: 334$

18. Isacsson U, Lu X (1999) J Mater Sci 34:3737. doi:10.1023/ A: 1004636329521

19. Lu X, Isacsson U, Ekblad J (1999) Mater Struct 32:131

20. Fawcett AH, McNally T (2000) Polymer 41:5315

21. Fawcett AH, McNally T (2001) Polym Eng Sci 41:1251

22. Fawcett AH, McNally T, McNally G (2002) Adv Polym Technol 21:275

23. Yousefi AA (2002) J Appl Polym Sci 90:3183

24. Gonzalez O, Munoz ME, Santamaria A, Garcia-Morales M, Navarro FJ, Partal P (2004) Eur Polym J 40:2365

25. Perez-Lepe A, Martinez-Boza FJ, Gallegos C (2005) Energy Fuels 19:1148

26. Polacco G, Muscente A, Biondi D, Santini S (2006) Eur Polym J 42:1113

27. Sengoz B, Isikyakar G (2008) Construct Build Mater 22:1897

28. Hesp SAM, Woodhams RT (1991) Colloid Polym Sci 269(8):825

29. Perez-Lepe A, Martinez-Boza FJ, Gallegos C (2007) J Appl Polym Sci 103:1166

30. Rojo E, Fernandez M, Pena JJ, Pena B, Munoz ME, Santamaria A (2004) Polym Eng Sci 44(9):1792

31. Gonzalez O, Munoz ME, Santamaria A (2006) Rheol Acta 45: 603

32. Hamielec AE, Soares JBP (1996) Prog Polym Sci 21:651

33. Liu C, Wang J, He J (2002) Polymer 43:3811

34. Masson J-F, Polomark G, Collins P (2005) Thermochim Acta 436:96

35. Mandelkern L, Garrett RR, Flory PJ (1952) J Am Chem Soc 74:3949

36. Lee H, Cho K, Ahn TK, Choe S, Kim IJ, Park I, Lee BH (1997) J Polym Sci B 35:1633

37. Lesueur D, Gérard JF, Claudy P, Létoffé JM, Martin D, Planche JP (1998) J Rheol 42:1059

38. Lesueur D, Gérard JF, Claudy P, Létoffé JM, Martin D, Planche JP (1996) J Rheol 40:813

39. Becker YM, Muller AJ, Rodriguez Y (2003) J Appl Polym Sci 90:1772 